\title{
Evaluating Dry Eye Symptoms After Phacoemulsification Using Ocular Surface Disease Index, Dry Eye Questionnaire-5, and Standardized Patient Evaluation of Eye Dryness
}

\author{
Vina Yuwanda*, Bobby R. E. Sitepu** \\ *Resident Medical Doctor, Mitra Medika Hospital \\ **Ophthalmologist in Department of Ophthalmology, Mitra Medika Hospital \\ DOI: 10.29322/IJSRP.11.08.2021.p11606 \\ http://dx.doi.org/10.29322/IJSRP.11.08.2021.p11605
}

\begin{abstract}
Introduction: This study aims to evaluate dry eye symptoms after phacoemulsification

Materials and methods: A prospective observational descriptive study was performed with total 80 eyes with cataracts in April 2021. All participants underwent phacoemulsification. All participants were asked Ocular Surface Disease Index (OSDI), Dry Eye Questionnaire-5 (DEQ-5), and Standardized Patient Evaluation of Eye Dryness (SPEED) preoperatively, 1 day, 1 week and 1 month after surgery.

Results: Eighty eyes out of 62 patients with senile cataract underwent phacoemulsification has mean age 63.2 years. Using Wilcoxon signed rank test, it showed that there were no significant differences between pre-surgery and a day after phacoemulsification (OSDI, $p=0.149$; SPEED, $p=0.354$; DEQ-5, $p=0.389$ ) or pre-surgery and a week (SPEED, $p=0.619$; DEQ-5, $p=0.965$ ) after phacoemulsification. Dry eye symptoms were related before and a week after phacoemulsification using OSDI questionnaire $(p=0.038)$. Significant difference a month after phacoemulsification $(p<0.00001)$. Dry eye symptoms are associated between pre-surgery and a month after phacoemulsification (OSDI, $p<0.00001$; SPEED, $\mathrm{p}=0.024$; DEQ$5, p<0.0003)$.

Conclusion: There was statistically difference in patients suffering dry eye symptoms before and a month after surgery using OSDI, SPEED and DEQ-5.
\end{abstract}

Index Terms - Dry Eye Symptoms, Ocular Surface Disease Index, Dry Eye Questionnaire-5, Standardized Patient Evaluation of Eye Dryness

\section{INTRODUCTION}

A cataract is an opacification of a lens that mostly happens in the elderly[1]. As it is the first leading cause of visual blindness and the second of visual impairment around the world, it decreases patients' quality of life[2], [3]. Cataract surgery aids in improving the quality of life by improving visual outcomes[4]. The phacoemulsification technique is most practiced in cataract surgery recently because of smaller incisions, faster healing time, less astigmatism[5], [6].
Dry Eye Disease is an ocular surface disease characterized by disequilibrium of the tear film and followed by ocular symptoms[7]. Tear film instability, hyperosmolarity, ocular surface inflammation and damage and neurosensory abnormalities cause feeling of grittiness, stinging, or burning sensations in the eyes[7], [8]. Other common risk factors that also cause dry eye disease are older age, female, computer use, contact lens, smoking, alcohol, oral contraceptives, diabetes, pterygium and medications (for example, antihistamine, diuretics, and $\beta$-blockers)[9].

Iatrogenic interventions, for instance, topical eye drops and cataract surgery, possibly cause tear film dysfunction and it persists for more than 3 months[10], [11]. Many patients who experienced cataract surgery complaining about having dry eye symptoms such as itchy, dry, and grittiness on the operated eye. Different technique of cataract surgery has different effects on dry eye disease. Incision on the corneal nerve results in slower epithelial wound healing and metabolic activity, increased epithelial permeability, deconstruction of cytoskeletal, and decreasing tear production that results in aqueous deficient of dry eye. Increasing inflammatory and chemical mediators irritate ocular surface structures that leads to hyper-evaporative of the tear film[12].

Tear Film and Ocular Surface Society (TFOS) recommends Dry Eye Questionnaire-5 (DEQ-5) and Ocular Surface Disease Index (OSDI) to assess dry eye disease. Standardized Patient Evaluation of Eye Dryness (SPEED) is also recommended because it can separate symptomatic and asymptomatic patients[13]. Other diagnostic tests are fluorescein break up test, osmolarity test and ocular surface staining[9]. These tests are uncomfortable and can stain around the eyes if not done properly. That's why we use questionnaires to evaluate dry eye in phacoemulsification in this research as phacoemulsification is the most used method in cataract surgery.

\section{MATERIALS AND METHODS}

A prospective observational study was performed from April to June 2021 in Mitra Medika Hospital located in Tanjung Mulia, Medan. A total of 62 patients with 80 eyes with cataracts underwent standard phacoemulsification in April are included in 
ISSN 2250-3153

this study. Patients with previous intraocular surgery on the same eye is excluded from the study.

\section{Surgery technique}

Patients underwent phacoemulsification and intraocular lens (IOL) implantation performed by the same single surgeon using bimanual microincision phacoemulsification. At first, tropicamide was given on operated eye 20 minutes before surgery. Under topical anesthesia, incisions were made. Intracameral anesthesia was injected. An ophthalmic viscosurgical device (OVD) (hydroxypropylmethyl cellulose 2\%) was injected into the anterior chamber. A clear corneal uniplanar incision was performed at 11 o'clock (superotemporal in right eyes and superonasal in left eyes) with a $2.75-\mathrm{mm}$ steel blade. After continuous curvilinear capsulorhexis, hydrodissection and hydrodelineation was performed, nucleus removal was done using stop and chop technique. OVD was injected to capsular bag, then a foldable monofocal posterior chamber IOL was inserted in the capsular bag. The incisions were closed with stromal hydration. Then, intracameral Ofloxacin was injected. After the surgery, patients used the same topical medication with ofloxacin, neomycin sulphate, Polymyxin B sulphate, and dexamethasone six times a day for the first week, then neomycin sulphate, Polymyxin B sulphate, and dexamethasone three times a day. Sodium chloride and potassium chloride lubricant eyedrop was used during the postoperative routinely. These eyedrops were preservative-free.

The OSDI questionnaire assesses subjective dry eye symptoms and quality of life-related to dry eye. There are 3 subscales with a total of 12 questions, asking about ocular symptoms, visual problems doing daily activities, and environmental triggers for a week. The answer to each question has five-point scales ranging from "None of the time" to "All of the time". Total scores range from 0 to 100 points, classified to normal (0-12 points) and dry eye symptoms (13-100 points)[14], [15].

The DEQ-5 is the short version of the Dry Eye Questionnaire (DEQ), which contains 5 questions, able to differentiate patients with and without dry eye and patients with Sjögren's syndrome and non-Sjögren's syndrome for a month. The severity of dry eye is assessed from "I don't have dry eye" has zero points to "Extremely severe" has 5 points. Points of $\geq 6$ indicate dry eye[16].

The SPEED questionnaire proposes 3 symptoms of dryness, burning and fatigue for the past 72 hours, a week, and 3 months. Cumulative scores are obtained by adding 5 responses to every 8 questions[17]. A cutoff point of 6 is considered for dry eye[18].

Ocular Surface Disease Index (OSDI), Standardized Patient Evaluation of Eye Dryness (SPEED), and Dry Eye Questionnaire5 (DEQ-5) were asked preoperatively, a day, a week, and a month postoperatively. The score of OSDI was collected by summing the scores for each question $\mathrm{x} 100 /$ total number of answered questions $\mathrm{x} 4$. Blurred vision and poor vision questions are excluded preoperatively since cataract also caused the same symptoms. Score of $\geq 13$ indicates dry eye symptoms. The SPEED questionnaire was earned by adding all the symptomatic scores. Total score of $\geq 6$ show dry eye symptoms[18]. Total score of more than 5 in DEQ-5 is considered having dry eye symptoms[16].

Statistical analysis was performed by IBM SPSS Statistics 25 . Dependent t-test or Wilcoxon signed-rank test was performed depends on the distribution. Quantitative results were shows as mean and its standard deviation. $\mathrm{P}$-value $<0,05$ were considered significant.

\section{RESULTS}

This study involved 80 eyes out of 62 patients with senile cataracts who went through phacoemulsification. There were 36 females $(58,07 \%)$ and 26 males $(41,93 \%)$. Their mean age was $63,25 \pm 7,278(\mathrm{SD})$ years, ranging from 45 to 87 years old.

Table 1. Characteristics of patients

\section{Characteristics}

\begin{tabular}{|l|l|c|}
\hline Sex & Female & 36 \\
\hline & Male & 26 \\
\hline & & Mean \\
\hline & & $63.25(45-87)$ \\
\hline
\end{tabular}

\section{Dry Eye Symptoms}

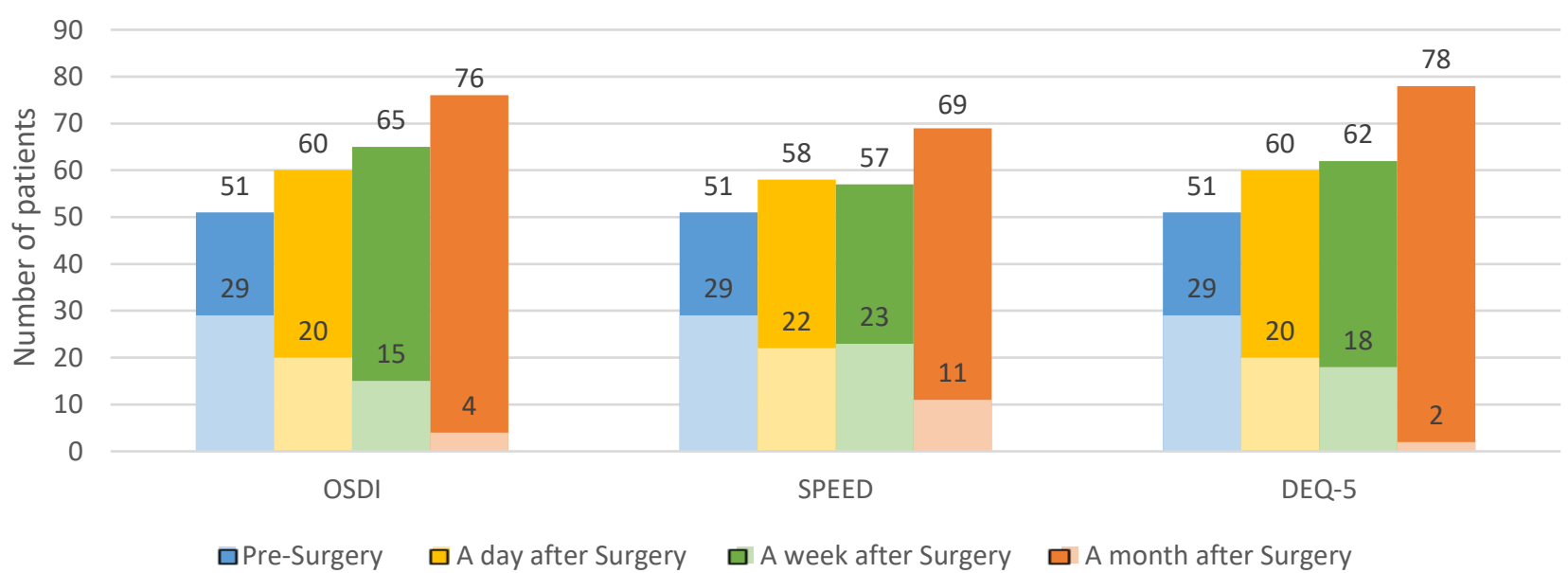

Figure 1. Number of patients having dry eye symptoms pre-surgery, a day, a week and a month after surgery using Ocular Surface Disease Index (OSDI), Standardized Patient Evaluation of Eye Dryness (SPEED) and Dry Eye Questionnaire 5 (DEQ-5).

This publication is licensed under Creative Commons Attribution CC BY. 


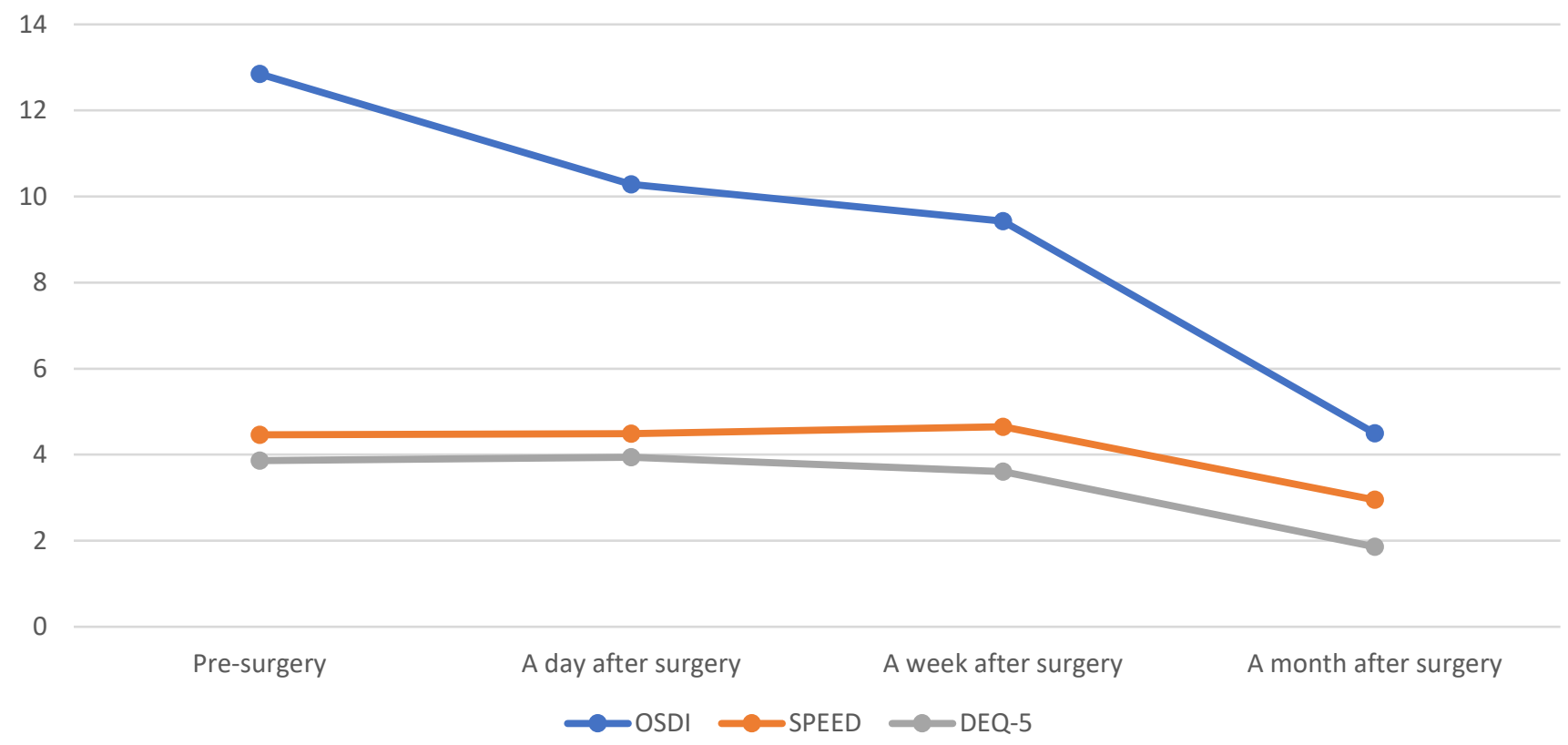

Figure 2. Mean score of Ocular Surface Disease Index (OSDI), Standardized Patient Evaluation of Eye Dryness (SPEED) and Dry Eye Questionnaire 5 (DEQ-5) that is taken before surgery, a day after surgery, a week after surgery and a month after surgery.

By using OSDI questionnaire, there were $29(36,3 \%)$ patients had dry eye symptoms and $51(63.7 \%)$ patients do not have dry eye symptoms preoperative. One day after surgery, 20 (25\%) patients had dry eye and 60 (75\%) patients are symptom-free. A week later, $15(18.8 \%)$ patients had dry eye symptoms and 65 $(81.3 \%)$ patients are symptom-free. A month after surgery, 4 (5\%) patients had dry eye symptoms and $76(95 \%)$ patients do not have dry eye symptom.

In SPEED questionnaire, there were 29 (36.3\%) patients had dry eye symptoms and $51(63.7 \%)$ patients do not have dry eye symptoms preoperative. One day after surgery, $22(27.5 \%)$ patients had dry eye and 58 (72.5\%) patients are symptom-free. A week later, $23(28.7 \%)$ patients had dry eye symptoms and 57 (71.3\%) patients are symptom-free. A month after surgery, 11 (13.8\%) patients had dry eye symptoms and $69(86.3 \%)$ patients do not have dry eye symptom.

In DEQ-5 questionnaire, there were 29 (36.3\%) patients had dry eye symptoms and $51(63.7 \%)$ patients do not have dry eye symptoms preoperative. One day after surgery, $20(25 \%)$ patients had dry eye and 60 (75\%) patients are symptom-free. A week later, $18(22.5 \%)$ patients had dry eye symptoms and $62(77.5 \%)$ patients are symptom-free. A month after surgery, 2 (2.5\%) patients had dry eye symptoms and $78(97.5 \%)$ patients do not have dry eye symptom.

The mean of OSDI score preoperatively, a day, a week, and a month were declining rapidly by the end of a month. SPEED

Table 2. Changes in dry eye questionnaires over postoperative time. questionnaire showed increasing in dry eye symptoms after a day and a week, then rapidly decreasing after a month. Using the DEQ-5 questionnaire, the score was increasing on day 1, then declining after a week and a month (Figure 2).

Mean and standard deviation of OSDI, SPEED and DEQ-5 showed that patients do not have dry eye before and phacoemulsification as shown in table 2. However, there were slightly increased of SPEED and OSDI score 24-hour after operation.

A Wilcoxon signed rank test was performed. By using OSDI questionnaire, it showed that there were no significant differences between pre-surgery and a day after phacoemulsification $(p=0.149)$. It showed significant difference between pre-surgery and a week $(p=0.038)$ after phacoemulsification or a month after phacoemulsification $(p<0.00001)$. Using SPEED questionnaire, significant difference was not found in a day $(p=0.354)$ and a week $(p=0.619)$ after phacoemulsification compare to before surgery, but significant difference was found a month $(\mathrm{p}=0.024)$ after phacoemulsification. By DEQ-5, there were no significant differences between pre-surgery and a day after phacoemulsification $(p=0.389)$ or pre-surgery and a week after phacoemulsification $(p=0.965)$. Dry eye symptoms are associated between pre-surgery and a month after phacoemulsification $(p<0.0003)$.

\begin{tabular}{|l|c|c|c|c|}
\hline & Pre-surgery & A day after surgery & A week after surgery & A month after surgery \\
\hline Mean OSDI score & $12.85 \pm 15.55$ & $10.28 \pm 9.41$ & $9.43 \pm 0.97$ & $4.50 \pm 0.53$ \\
\hline Mean SPEED score & $4.46 \pm 5.42$ & $4.49 \pm 3.67$ & $4.65 \pm 3.59$ & $2.95 \pm 2.59$ \\
\hline Mean DEQ-5 score & $3.86 \pm 4.25$ & $3.94 \pm 3.42$ & $3.61 \pm 3.23$ & $1.86 \pm 1.75$ \\
\hline
\end{tabular}

This publication is licensed under Creative Commons Attribution CC BY. 
Table 3. Correlations between pre-surgery and after surgery using Ocular Surface Disease Index (OSDI), Standardized Patient Evaluation of Eye Dryness (SPEED) and Dry Eye Questionnaire 5 (DEQ-5).

\begin{tabular}{|c|c|c|c|c|}
\hline \multicolumn{2}{|c|}{$\boldsymbol{p}$-value } & $\begin{array}{r}\text { A day After } \\
\text { Phacoemulsification }\end{array}$ & $\begin{array}{r}\text { A week After } \\
\text { Phacoemulsification }\end{array}$ & $\begin{array}{c}\text { A month After } \\
\text { Phacoemulsification }\end{array}$ \\
\hline \multirow{3}{*}{ Pre-Surgery } & OSDI & 0.149 & 0.038 & 0.00001 \\
\cline { 2 - 5 } & SPEED & 0.354 & 0.619 & 0.024 \\
\cline { 2 - 5 } & DEQ-5 & 0.389 & 0.965 & 0.0003 \\
\hline
\end{tabular}

\section{IV.DISCUSSION}

Dry eye disease is an ocular surface disease that has instability of tear film and followed by ocular symptoms[9]. Prevalence of dry eye disease ranging from $5 \%$ to $30 \%$ in various age groups and countries[19]. Incidence rates of dry eye of people around 43 and 86 years old for 5 and 10 years of research were $13.3 \%$ and $21.6 \%$ [20]. Prevalence of dry eye disease was $22.4 \%$ in elderly patients with cataract in Jakarta, Indonesia[21]. Study from Kanfade et al showed that the incidence of dry eye after small incision cataract surgery (SICS) was 73\%.[22] Another study by Ayrika, et al showed that there were $92.9 \%$ had dry eye disease after extracapsular cataract extraction (ECCE) technique; $21.4 \%$ after SICS; and 32.1\% after phacoemulsification [23].

This study reported that there were vaguely increasing of dry eye symptoms in one day after phacoemulsification. However, the mean scores of each questionnaire did not meet dry eye disease classification.

Systemic risk factors for dry eye disease were Sjögren syndrome, androgen deficiency, diabetes, rosacea, thyroid disease, pterygium, refractive surgery, menopause and sarcoidosis. Smoking, alcohol and oral contraceptives also caused dry eye disease [9].

A study by Qayum and Iqbal demonstrated the incidence of dry eye after phacoemulsification was $11 \%$ and the highest score using OSDI questionnaire was at a week after surgery. It was reported, before surgery, the mean score was 13.43 , followed by 13,43 one day after surgery, significantly higher, 25.98, after one week and 22.14 after one month [24].

In this study, OSDI scores after surgery were decreasing compare to prior to surgery. Higher mean score of OSDI (12.8\%) preoperative could be possibly caused by a few patients having cortical cataract that caused photosensitivity.

Study from Favuzza, et al. showed that the mean score of SPEED was increased one week after phacoemulsification. Preoperative SPEED score was $1.68 \pm 1.47$, one week later it was $2.19 \pm 1.82$, and one month postoperative, it was $1.63 \pm 1.82$ [25]. Similar result was shown in this study, where the mean SPEED score was highest at one week after surgery.

Another study using DEQ-5 by Recchioni, et al. presented increasing dry eye symptoms after 1 month phacoemulsification by $21 \%$ [26]. It was different compared to this study, which was decreasing symptoms in a month. Different results could be possibly because of small number of participants by the previous study $(n=41)$ causing lack of variation.

It is common that cataract surgery caused dry eye [27]. Cornea has the most innervated organ, supplied by sensory neurons of trigeminal nerve. Sensory fibers were from nasociliary branch of

This publication is licensed under Creative Commons Attribution CC BY. ophthalmic nerve and long ciliary nerves. Pericorneal plexus was made of long and short ciliary nerves at corneal limbus. Corneal nerve fibers dispersed to stromal and subepithelial plexus in corneoscleral limbus. Facial nerves had motor and autonomic fibers which controlled blinking and stimulated tear secretion from lacrimal gland. Sensory nerve endings could change the intensity of tear production by trigeminal-parasympathetic reflex depends on environmental changes. Neural response mechanisms regulated secretion by goblet cells and meibomian glands. Reduced tear secretion causing thinner tear film and that leaded abnormal structure of corneal and conjunctival nerve fibers [28]. Cataract surgery often triggered production of inflammatory cytokines, interleukins and proteases which caused apoptotic of surface epithelial cells. Apoptotic cells promoting more inflammatory process causing a repeating cycle [29]. These inflammations causing ocular surface epithelial cells metaplasia and leaded to loss of goblet cells and reduced expression of glycocalyx mucins [29], [30]. Physiological decreased production of lacrimal glands in elderly, it will aggregate hyperosmolarity of ocular surface [30].

Pathophysiology of dry eye disease could be predisposed prior to operation, intraoperation and post-operation. Preoperative events that may lead to ocular surface disease were topical anesthetics and mydriatics [30]. Topical anesthetics for example tetracaine $0.5 \%$ could damage cell membrane, loss of microvilli and desquamation of corneal epithelium[31]. Topical mydriatics may cause blepharitis and epithelial desquamation of cornea. Adding phenylephrine 10\% may cause loosing of iris pigment [30], [31]. Preservative usage may result in corneal and conjunctival epithelial cell toxicity, tear film instability and corneal epithelial barrier dysfunction.

During operation, incision size and location, light exposure from microscope and thermal energy from phacoemulsification device might cause dry eye disease [32]. After phacoemulsification, corneal sensitivity was impaired, which influenced tear production [32]. Study from Ishrat et al. reported that there was higher prevalence of dry eye disease in patients underwent SICS $(6-7 \mathrm{~mm})$ than phacoemulsification $(2.75 \mathrm{~mm})$ because of greater corneal denervation that resulted in reduced tear secretion, less blinking, persistent foreign body sensation and pooling of mucus within the fissure [27]. A research from Suryani et al. showed that temporal incision induced more dry eye symptoms than superior incision on 30 days after phacoemulsification [33]. Light exposure can trigger Interleukin$1 \beta$, that promote the production of reactive oxygen species (ROS). Thus, longer operative time, the more damage on the ocular surface and goblet cell density [30]. Study from Garg et al. proved that operative time $>15$ minutes was related to increased risk of dry eye, reduced tear break up time [34]. Phacoemulsification 
ISSN 2250-3153

devices generated thermal energy hence longer phacoemulsification time damages the corneal nerve [12], [32].

It is recommended to use artificial tears before and after surgery. A study showed that the mean score of SPEED questionnaire did not show any significant changes in patients using artificial tears before and after surgery, but worsened in untreated patients[25]. In this study, all patients used artificial tears, antibiotics and anti-inflammatory drugs postoperative.

\section{CONCLUSION}

Dry eye disease is caused by tear hyperosmolarity, tear instability and inflammation. Cataract surgery, for example phacoemulsification, can cause dry eye symptoms. Tetracaine eye drops, surgical time, light exposure, corneal incision as the risk factor for dry eye disease during phacoemulsification. Dry eye symptoms were evaluated by using OSDI, SPEED and DEQ-5. These questionnaires showed declining numbers of patients having dry eye symptoms after a week. It was slightly increased at the first week, because of corneal damage during phacoemulsification. These questionnaires were recommended to be used as screening tools for dry eye disease. The use of hemostasis markers, for instance, tear breakup time, osmolarity and ocular surface staining, could make patients felt uncomfortable and chaotic. To prevent dry eye symptoms and increasing patients' quality of life, the use of antibiotics, antiinflammatory drug and lubricants were recommended.

\section{References}

1. Liu, Y. C., Wilkins, M., Kim, T., Malyugin, B. \& Mehta, J. S. Cataracts. Lancet 390, 600-612 (2017).

2. Flaxman, S. R. et al. Global causes of blindness and distance vision impairment 1990-2020: a systematic review and meta-analysis. Lancet. Glob. Heal. 5, e1221-e1234 (2017).

3. Hashemi, H. et al. Global and regional estimates of prevalence of refractive errors: Systematic review and meta-analysis. J. Curr. Ophthalmol. 30, 3-22 (2018).

4. Yoshizaki, M. et al. Interventions to improve the quality of cataract services: protocol for a global scoping review. BMJ Open 10, e036413 (2020).

5. Gharaee, H., Kargozar, A., Daneshvar-Kakhki, R., Sharepour, M. \& Hassanzadeh, S. Correlation between Corneal Endothelial Cell Loss and Location of Phacoemulsification Incision. J. Ophthalmic Vis. Res. 6 , 13-17 (2011).

6. Saad Filho, R. et al. Costs and outcomes of phacoemulsification for cataracts performed by residents. Arq. Bras. Oftalmol. 83, 209-214 (2020).

7. Wolffsohn, J. S. et al. TFOS DEWS II Diagnostic Methodology report. Ocul. Surf. 15, 539-574 (2017).

8. Dana, R., Meunier, J., Markowitz, J. T., Joseph, C. \& Siffel, C. PatientReported Burden of Dry Eye Disease in the United States: Results of an Online Cross-Sectional Survey. Am. J. Ophthalmol. 216, 7-17 (2020).

9. Craig, J. P. et al. TFOS DEWS II Report Executive Summary. Ocul. Surf 15, 802-812 (2017).

10. Jiang, D. et al. Transient tear film dysfunction after cataract surgery in diabetic patients. PLoS One 11, 1-8 (2016).

11. Xue, W. et al. Long-term impact of dry eye symptoms on vision-related quality of life after phacoemulsification surgery. Int. Ophthalmol. 39, 419-429 (2019).

12.

Sutu, C., Fukuoka, H. \& Afshari, N. A. Mechanisms and management of dry eye in cataract surgery patients. Curr. Opin. Ophthalmol. 27, 24-30 (2016).

13. Asiedu, K. et al. Ocular surface disease index (OSDI) versus the standard patient evaluation of eye dryness (SPEED): A study of a nonclinical sample. Cornea 35, 175-180 (2016).

14. Okumura, Y. et al. A review of dry eye questionnaires: Measuring patient-reported outcomes and health-related quality of life. Diagnostics
10, $1-21(2020)$

15. Barber, L. et al. Dry eye symptoms and impact on vision-related function across International Task Force guidelines severity levels in the United States 11 Medical and Health Sciences 1113 Ophthalmology and Optometry. BMC Ophthalmol. 18, 1-9 (2018).

16. Chalmers, R. L., Begley, C. G. \& Caffery, B. Validation of the 5-Item Dry Eye Questionnaire (DEQ-5): Discrimination across self-assessed severity and aqueous tear deficient dry eye diagnoses. Contact Lens Anterior Eye 33, 55-60 (2010).

17. Ngo, W. et al. Psychometric properties and validation of the standard patient evaluation of eye dryness questionnaire. Cornea 32, 1204-1210 (2013).

18. Asiedu, K., Kyei, S., Boampong, F. \& Ocansey, S. Symptomatic Dry Eye and Its Associated Factors: A Study of University Undergraduate Students in Ghana. Eye Contact Lens 43, 262-266 (2017).

19. Phadatare, S. P., Momin, M., Nighojkar, P., Askarkar, S. \& Singh, K. K. A Comprehensive Review on Dry Eye Disease: Diagnosis, Medical Management, Recent Developments, and Future Challenges. Adv. Pharm. 2015, 704946 (2015).

20. Kasetsuwan, N., Satitpitakul, V., Changul, T. \& Jariyakosol, S. Incidence and pattern of dry eye after cataract surgery. PLoS One $\mathbf{8}$, e78657 (2013).

21. Noor, N. A., Rahayu, T. \& Gondhowiardjo, T. D. Prevalence of dry eye and its subtypes in an elderly population with cataracts in Indonesia. Clin. Ophthalmol. 14, 2143-2150 (2020).

22. Ekanksha Kanfade, Sachin Daigavane, Sonal Muley, C. S. A Prospective Study of Dry Eye in Patients after Manual Small Incision Cataract Surgery. Indian J. Forensic Med. \&amp; Toxicol. 14, 6340$6346(2020)$.

23. Yuliani, A. \& Siti Harilza Z, T. Relationship Between Dry Eyes Syndrome With Cataract Surgical Techniques In Patients After Cataract Surgery At H. Adam Malik Hospital Medan And Network. Int. J. Sci. Res. Publ. 8, 704-707 (2018).

24. Qayum, D. S. Incidence of Dry eye after clear corneal phacoemulsification. J. Med. Sci. Clin. Res. 7, 964-969 (2019).

25. Favuzza, E., Cennamo, M., Vicchio, L., Giansanti, F. \& Mencucci, R. Protecting the ocular surface in cataract surgery: The efficacy of the perioperative use of a hydroxypropyl guar and hyaluronic acid ophthalmic solution. Clin. Ophthalmol. 14, 1769-1775 (2020).

26. Recchioni, A., Donnell, C. O. \& Wolffsohn, J. S. W. The role of dry eye disease in cataract and refractive surgery. (2019).

27. Ishrat, S., Nema, N. \& Chandravanshi, S. C. L. Incidence and pattern of dry eye after cataract surgery. Saudi J. Ophthalmol. 33, 34-40 (2019).

28. Mikalauskiene, L., Grzybowski, A. \& Zemaitiene, R. Ocular Surface Changes Associated with Ophthalmic Surgery. J. Clin. Med. 10, (2021).

29. Kohli, P., Arya, S. K., Raj, A. \& Handa, U. Changes in ocular surface status after phacoemulsification in patients with senile cataract. Int. Ophthalmol. 39, 1345-1353 (2019).

30. Mencucci, R. et al. Iatrogenic Dry Eye Disease: Dealing with the Conundrum of Post-Cataract Discomfort. A P.I.C.A.S.S.O. Board Narrative Review. Ophthalmol. Ther. 10, 211-223 (2021).

31. Hopkins, G. \& Pearson, R. Local Anaesthetics. in Ophthalmic Drugs 139-147 (Elsevier Ltd, 2007). doi:10.1016/b978-0-7506-8864-2.500167.

32. Dhawan, M. Dry Eye After Phacoemulsification. Delhi J. Ophthalmol. 29, (2018).

33. Suryani, Syawal Rukiah, S. \& Hamzah. The Comparison Of Dry Eye Between The Position Of The Temporal Incision With The Position Of The Superior Incision After Phacoemulsification. (2017).

34. Garg, P., Gupta, A., Tandon, N. \& Raj, P. Dry eye disease after cataract surgery: Study of its determinants and risk factors. Turkish J. Ophthalmol. 50, 133-142 (2020).

\section{AUTHORS}

First Author - Vina Yuwanda, Department of Ophthalmology, Mitra Medika Hospital. vinayuwanda@outlook.com

Second Author - Bobby R.E. Sitepu, Department of Ophthalmology, Mitra Medika Hospital, bobbyres@yahoo.com Correspondence Author - Vina Yuwanda, vinayuwanda@outlook.com, +6285761247236

This publication is licensed under Creative Commons Attribution CC BY. 
